\title{
Hemilaminectomy approach for intradural extramedullary spinal tumors: an analysis of 164 patients
}

\author{
Mazda Keki Turel, MCh, Wilson Prashanth D’Souza, MBBS, and Vedantam Rajshekhar, MCh \\ Department of Neurological Sciences, Christian Medical College, Vellore, Tamil Nadu, India
}

OBJECT The authors report their experience with the use of a minimally invasive hemilaminectomy approach for the excision of intradural extramedullary (IDEM) tumors of the spinal cord.

METHODS Included were 164 consecutive patients (167 tumors in total), treated between January 2004 and December 2014, in whom the hemilaminectomy approach for IDEM spinal cord tumors was planned. The clinicoradiological data of patients presenting to Christian Medical College were analyzed retrospectively using a computerized chart-review method. Preoperative MRI localization was used in 51 cases.

RESULTS The mean age ( \pm SD) of the patients was $42.6 \pm 16.6$ years, and the mean duration of symptoms was 20.8 \pm 27.8 months. The average tumor size was $2.4 \pm 1.7 \mathrm{~cm}$. Forty-four tumors were cervical, 5 were cervicothoracic, 72 were thoracic, 7 were thoracolumbar, and 39 were lumbar. Forty-two tumors were ventrally located, whereas 11 were ventrolateral; the rest were either dorsal or dorsolateral. The most common pathologies were schwannoma (110 cases) and meningioma (31 cases). Total excision was achieved for $93 \%$ of the tumors. The average duration of surgery was 1.6 \pm 0.6 hours.

CONCLUSIONS The hemilaminectomy approach to the removal of intradural tumors was quick and safe and resulted in minimal morbidity and no mortality. Preoperative MRI localization improved accuracy and reduced operating time by eliminating the need for intraoperative fluoroscopy.

http://thejns.org/doi/abs/10.3171/2015.5.FOCUS15170

KEY WORDS hemilaminectomy; extramedullary; intradural; spine; tumor

I NTRADURAL extramedullary (IDEM) tumors account for two-thirds of spinal cord tumors. ${ }^{7}$ Although schwannomas and neurofibromas are more common in the cervical spine and lumbar spine, meningiomas predominate in the thoracic spine. ${ }^{2}$ Because IDEM tumors are almost always benign, total excision should be the goal of surgical treatment to provide a cure to the patient. The traditional approach to excising these tumors involves a laminectomy at the involved levels for adequate exposure. Although this approach has stood the test of time, it is associated with significant tissue trauma and blood loss and long hospital stays, especially when multiple levels are involved. ${ }^{5}$ With the advent of minimally invasive procedures, more emphasis is being placed on curtailing the amount of bone and ligament removal to reduce postoperative back pain, mini- mize blood loss, shorten the duration of hospital stay, and eliminate the risk of possible instability while achieving the same desired result. In this article, we describe our experience with a large series of patients in whom hemilaminectomy/laminotomy was used for these tumors, and we discuss the advantages and disadvantages of this technique.

\section{Methods}

One hundred sixty-four consecutive patients with a preoperative diagnosis of an IDEM tumor (167 tumors in total) and an operative plan for minimally invasive hemilaminectomy were included in this study between January 2004 and December 2014. The size, laterality, number of segments involved, and probable pathologies were criteria

ABBREVIATIONS IDEM = intradural extramedullary; MEP = motor evoked potential; MMEP = myogenic MEP.

SUBMITTED March 31, 2015. ACCEPTED May 14, 2015.

INCLUDE WHEN CITING DOI: 10.3171/2015.5.FOCUS15170.

DISCLOSURE The authors report no conflict of interest concerning the materials or methods used in this study or the findings specified in this paper. 
for deciding on the hemilaminectomy approach. The patients' clinicoradiological features were entered retrospectively into a database (Microsoft Excel). For patients with a cervicothoracic pathology, Nurick grading was used to determine outcomes, and for those with a lumbar pathology, we assessed the severity of their pain. Each patient underwent preoperative Gd-enhanced MRI. In the initial 113 cases, the level of surgery for thoracic and upper-lumbar tumors was determined after examination of a preoperative marker radiograph the evening before surgery. In the last 51 cases we used T2-weighted MR images with placement of cod liver oil capsules on the patient's back to determine the appropriate level. For localizing cervical tumors, clinical palpation of the C-2 or C-7 vertebra served as an appropriate guideline. Most of the patients underwent surgery in the prone position, but some patients with C1-2 tumors located ventrolaterally underwent surgery in the lateral position. Patients were usually discharged on the 2nd or 3rd day after surgery. MRI was performed 3-12 months after surgery to determine the extent of excision.

\section{Electrophysiological Monitoring}

We used myogenic motor evoked potential (MMEP) monitoring for all the patients; we did not use somatosensory evoked potential monitoring or electromyography. MEP recording was performed by using transcranial electrical stimulation of the motor cortex. The stimulating electrodes were placed on the scalp (in either the hand or the leg area), and the recording electrodes were placed in the belly of various muscles in the lower limbs (tibialis anterior, quadriceps, soleus, extensor digitorum brevis) and the external anal sphincter. The stimulating current varied from a minimum of $100 \mathrm{~V}$ to a maximum of $800 \mathrm{~V}$. MEPs were obtained during surgery by epidural recording from either the spinal cord (D and I waves) or the muscles in the limbs (MMEPs). Although D-wave recordings are more robust and not affected by anesthesia, they do not provide information about specific muscle groups; hence, we prefer to use MMEPs. However, the results of the MEP monitoring and root stimulation in this study are being analyzed separately and will be presented in a separate communication.

\section{Algorithm for Deciding on Minimally Invasive Surgery}

The decision to use a unilateral hemilaminectomy approach was based entirely on radiological data. A minimally invasive approach was planned for each tumor that on imaging was undoubtedly intradural and extramedullary, even if it had an extradural/extraforaminal extension, provided it was not $>3$ vertebral bodies long. It is our experience that, if studied carefully, the radiological data will reveal that these tumors are almost always eccentric, and the laterality of the tumor guides us in choosing the side of the approach. However, one should always be prepared to abandon the minimally invasive approach for the traditional approach if decompression of the tumor is not possible because of its consistency, if the tumor has a poor plane with the spinal cord, or if, at surgery, the tumor is discovered to be an exophytic intramedullary tumor that mimicked an extramedullary tumor on imaging. Radiol- ogy results consistent with an intramedullary tumor ruled out use of the hemilaminectomy approach.

\section{Surgical Technique}

Preoperative steroids (dexamethasone $12-16 \mathrm{mg} /$ day) were administered from 1 day before surgery to 3 days after surgery. Localization of the levels involved was done with a marker radiograph/MR image acquired the night before surgery. In the operating room, after being intubated, the patient was turned prone (except for some patients with a ventral C-1 or C-2 tumor, who were placed in the lateral position). A small midline skin incision was made based on the markings made the day before, and dissection was carried out to expose the unilateral hemilamina or laminae. The supraspinal ligaments, interspinal ligaments, and tendinous insertions of the contralateral muscles were retained. A perpendicular trajectory was maintained by removing bone and ligaments to expose the dura on the side of the lesion. This was done by using highspeed drills (Aesculap), initially with a steel bur and then with a diamond bur, as the dural tube was approached. Kerrison rongeurs were used to remove the soft tissue and the ligamentum flavum to expose the dural tube. The dura was opened. The arachnoid was cut to mobilize the tumor. Because of the small exposure, after multiple pieces were taken for histopathology, tumors were almost always initially decompressed internally by using a Cavitronic ultrasonic aspirator (CUSA EXcel, Integra, Inc.). This, we believe, is a key step in the excision of these tumors. No attempt should be made to dissect outside of the tumor until it has been cored out from within and only a thin rim of the tumor capsule is left. Trying to deliver the tumor without reducing its bulk can result in injury to the adjacent normal neural structures. In each case of schwannoma, the affected root was usually coagulated and cut.

For some ventrally located tumors, the dural incision was extended in a T-shaped manner after performing a partial facetectomy on the involved side to enable removal of the tumor without any manipulation of the cord. The dentate ligaments were sectioned to achieve adequate visualization. There is almost never a need to instrument the spine, because the ribs provide adequate stability even when a facetectomy is performed on 1 side in the thoracic spine. For tumors with an extraforaminal extension, a partial facetectomy was performed, which enabled us to follow the tumor along the neural foramen. No instrumentation was used for stabilization in any of the patients. For meningiomas, the involved dura was either excised or, most often, coagulated completely. After the tumor excision was performed using an operating microscope, the dura was closed primarily with 7-0 Proline (Ethicon, Inc.). Differently sized needles are available for the same size of suture, and it is important to choose the correct one. We used 8-mm needles that were three-eighths of a circle, which do not make large dural holes but allow for easy maneuverability in this narrow space. For cases in which a primary closure was not possible, a fat graft was layered over the defect and covered with gelatin sponge. Patients ambulated within a few hours after surgery and were usually discharged on the 2 nd or 3 rd postoperative day. Patients with meningiomas in whom the dural defect 
could not be repaired were placed on bed rest in the prone position with a wound drain in place for 5 days. They were subsequently mobilized, and in most cases, we were able to avoid a CSF leak by using this technique.

\section{Results \\ Clinicoradiological Outcomes}

We included 164 patients (167 tumors in total) who underwent surgery between 2004 and 2014. There was a male preponderance (109 male, 55 female) with a mean age $( \pm$ SD) of $42.6 \pm 16.6$ years and a mean duration of symptoms of $20.8 \pm 27.8$ months. Tumor sizes ranged from 1.5 to $6 \mathrm{~cm}$ (average tumor size $2.4 \pm 1.7 \mathrm{~cm}$ ). The locations of the tumors within the spine and their respective pathologies are listed in Table 1. Twenty-three of these tumors had some form of extradural extension, and 8 had an extraforaminal extension. Of the meningiomas, 17 (55\%) were ventral or ventrolateral and 14 were dorsal or dorsolateral. Of the 110 schwannomas, $33(30 \%)$ were ventral or ventrolateral, and the rest were dorsal.

Three patients had a postoperative CSF leak; 2 of the leaks resolved by reapplying a suture, and 1 required insertion of a lumbar subarachnoid drain for the leak to subside.

\section{TABLE 1. Patient and tumor data*}

\begin{tabular}{|c|c|}
\hline Variable & Value (range) \\
\hline \multicolumn{2}{|l|}{ Patient data } \\
\hline Patients (total) & 164 \\
\hline Male & 109 \\
\hline Female & 55 \\
\hline Age (yrs) & $42.6 \pm 16.6(4-80)$ \\
\hline Symptom duration (mos) & $20.8 \pm 27.8(0.5-120)$ \\
\hline \multicolumn{2}{|l|}{ Tumor data } \\
\hline Tumors (total) & 167 \\
\hline Size $(\mathrm{cm})$ & $2.4 \pm 1.7(1.5-6)$ \\
\hline \multicolumn{2}{|l|}{ Location } \\
\hline Cervical & 44 \\
\hline Cervicothoracic & 5 \\
\hline Thoracic & 72 \\
\hline Thoracolumbar & 7 \\
\hline Lumbar & 39 \\
\hline \multicolumn{2}{|l|}{ Pathology } \\
\hline Schwannoma & 110 \\
\hline Meningioma & 31 \\
\hline PNET/sarcoma & 5 \\
\hline Neurofibroma & 3 \\
\hline Epidermoid cyst & 3 \\
\hline Paraganglioma & 3 \\
\hline Ependymoma & 3 \\
\hline Cavernoma/hemangioma & 3 \\
\hline Other & 6 \\
\hline
\end{tabular}

PNET = primitive neuroectodermal tumor.

* Values are expressed as the mean \pm SD (range) or number of patients or tumors.

\section{Surgical Outcomes}

The average duration of surgery was $1.6 \pm 0.6$ hours. We achieved total excision in all $(92 \%)$ but 13 of the tumors (Figs. 1 and 2). Of the tumors for which subtotal excision was performed, 5 were schwannomas in which the extraspinal extension was partially removed, 4 were meningiomas with some residue, 2 were sarcomas, 1 was an epidermoid cyst in which the capsule adherent to the conus medullaris was left behind, and 1 was an endodermal cyst that was marsupialized, which left behind the capsule adherent to the pia mater. In 5 cases (all involving the thoracic spine), the hemilaminectomy had to be extended 1 level above or below the planned level because of improper visualization of the tumor. However, 4 of these extensions were made when we used radiography for localization and only 1 was made when we used MRI with cod liver oil capsules for localization. This process, too, was standardized later by maintaining the same position of the patient's arms in the operating room as in preoperative MRI performed the night before surgery.

In 1 case, the hemilaminectomy had to be converted into a laminectomy. There was a dorsal meningioma with ossified dorsal dura for which we had to change the plan to enable excision of the involved dura.

\section{Follow-Up Results}

Of the 167 tumors in 164 patients, 121 (73.8\%) were treated with cervical or cervicothoracic hemilaminectomy and $46(28 \%)$ with thoracolumbar/lumbar hemilaminectomy. Of the 121 cervical or cervicothoracic IDEM tumors, spasticity had improved at discharge in $97(80 \%)$ of the cases and remained unchanged in $19(15.7 \%)$. There was a slight worsening in spasticity/power in 5 (4.1\%). Of these 5 patients with worsened spasticity/power at discharge, more than 3 months of follow-up data were available for 3 patients, all of whom showed significant improvement in spasticity and a return of normal power. Two patients had a pseudomeningocele, which did not require additional surgery.

Of the 46 lumbar IDEM or intradural tumors, back pain or radiculopathy had improved at discharge in 35 (76\%) of the cases, remained the same as the preoperative state in $9(20 \%)$, and worsened in $2(4 \%)$. The 2 patients who had worsening radiculopathy in the immediate postoperative period showed marginal improvement at discharge and were asymptomatic at follow-up.

We had long-term follow-up data for $115(71 \%)$ of the 164 patients (mean duration [ \pm SD] of follow-up $20.5 \pm$ 18.6 months; range 3-98 months). One hundred patients had at least 6 months of follow-up (Figs. 1 and 2). The mean preoperative and postoperative Nurick grades at last follow-up in patients with cervicothoracic tumors were 3.2 \pm 1.2 and $1.0 \pm 1.3(\mathrm{p}<0.05)$, respectively. No patient with a lumbar tumor had a neurological deficit at follow-up.

\section{Discussion}

\section{Preoperative Localization of Tumors}

Accurate intraoperative localization of small IDEM spinal tumors is essential, especially when minimally invasive approaches, such as hemilaminectomy, are used for 

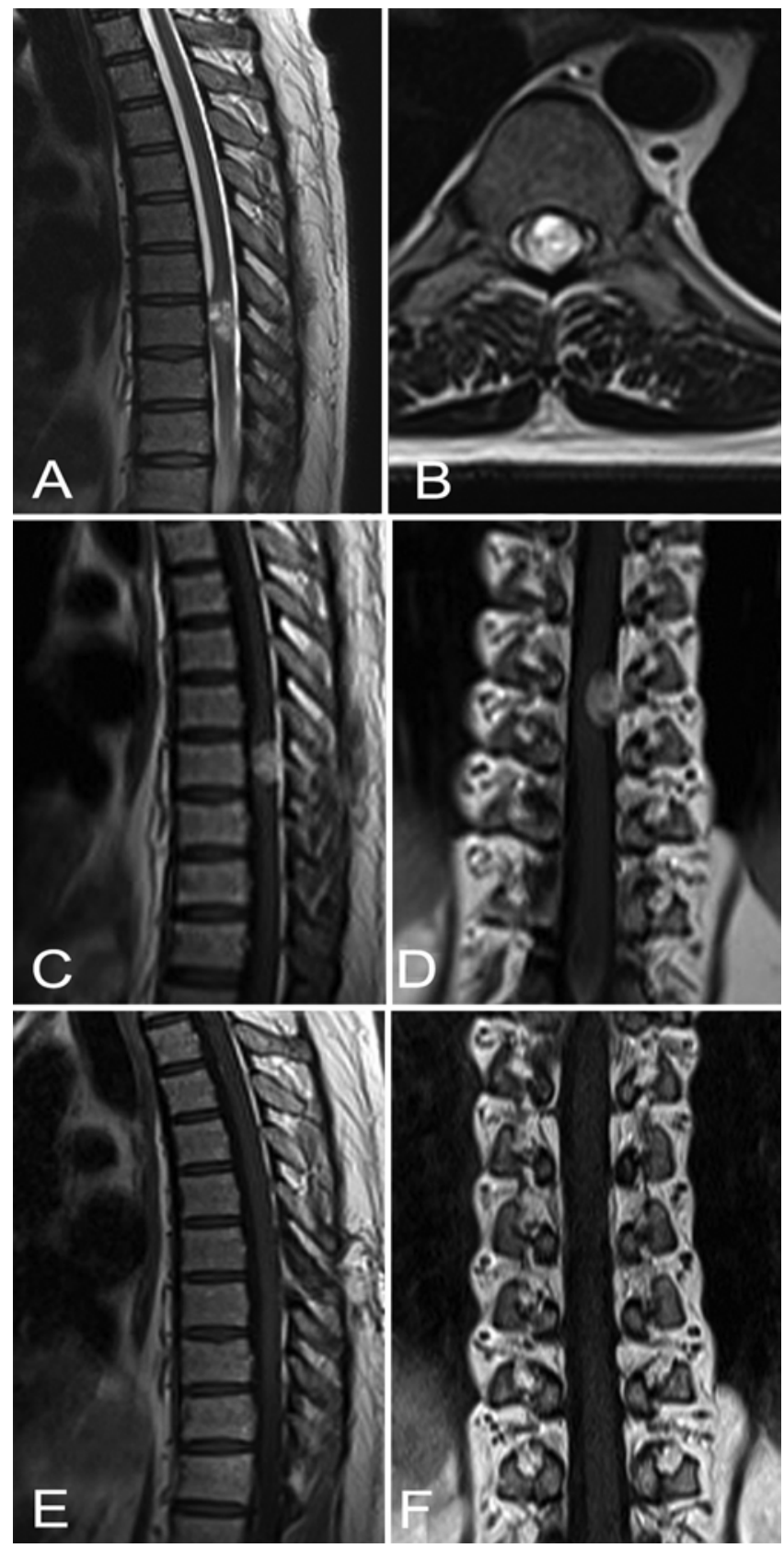

FIG. 1. Preoperative T2-weighted sagittal (A) and axial (B) MR images and sagittal (C) and coronal (D) Gd-enhanced MR images of a 50-yearold man who presented with back pain and numbness in both lower limbs. The images show a T2 hyperintense, $1.9-\mathrm{cm}$ dorsally located IDEM tumor at T-8, more to the left side, displacing the spinal cord to the right. We performed a T-8 left hemilaminectomy and achieved total excision of the schwannoma. One-year postoperative $\mathrm{Gd}$-enhanced T1-weighted sagittal $(E)$ and coronal $(F)$ images show no residual tumor and minimal soft-tissue damage resulting from the approach used. The patient made an excellent recovery and experienced improvement in his lower-limb sensations and no residual back pain.

the excision of these lesions. Although in the cervical and lower lumbar region this difficulty can be overcome easily, most errors occur in the thoracic and upper lumbar segments. Several invasive and noninvasive methods for appropriate localization have been described, along with
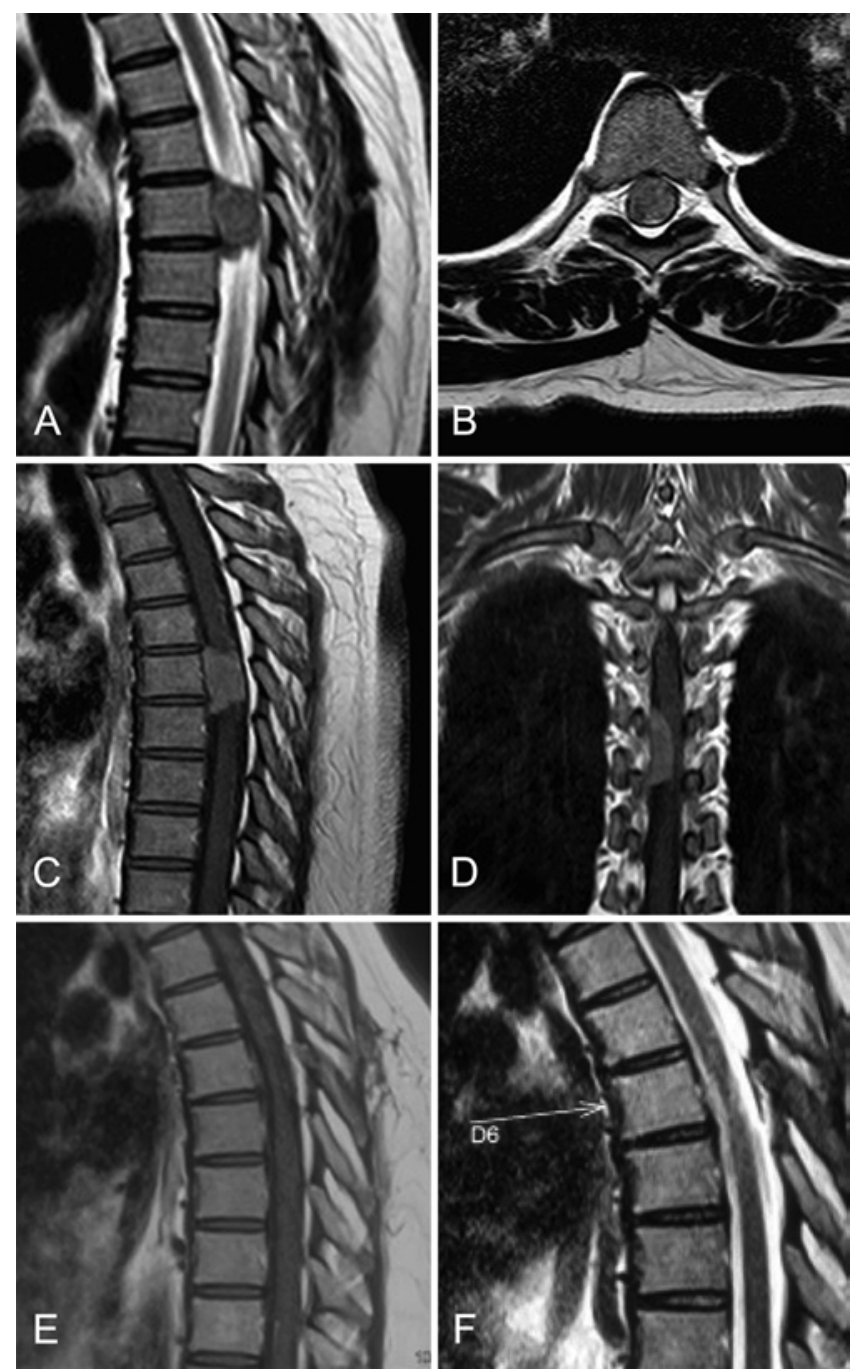

FIG. 2. Preoperative T2-weighted sagittal (A) and axial (B) MR images and sagittal (C) and coronal (D) Gd-enhanced MR images of a 44-year-old woman who had a Nurick Grade 4 tumor at presentation. The images show a T2 hypointense, $2.3-\mathrm{cm}$ ventrally located IDEM tumor, more to the right side, displacing the spinal cord to the left. The broad-based dural attachment to the ventrolateral dura is apparent. We performed a T-6 left hemilaminectomy and achieved total excision of the meningioma. One-year postoperative Gd-enhanced T1-weighted sagittal (E) and T2-weighted sagittal (F) MR images show no residual tumor and minimal soft-tissue changes. The patient made an excellent recovery and had a Nurick grade of 1 at follow-up.

their advantages and disadvantages, in the literature. ${ }^{3,10,12}$ We recently described the use of cod liver oil capsules in the preoperative MRI localization of intradural tumors, which resulted in a 97\% accuracy rate, and we feel comfortable using this technique. Our method of localization is safe and accurate and prevents the need for intraoperative fluoroscopy because it is based on the principle of visualizing the intradural pathology, which eliminates the need to correlate the level with the bony anatomy. On the evening before surgery, we placed 9 capsules in 3 rows at the level of the lesion and performed MRI with the patient in the prone position. ${ }^{13}$ 


\section{Electrophysiological Monitoring}

We did not use somatosensory evoked potential monitoring for these tumors, because we found MEP monitoring reliable. In our institution, MEPs have been found to show good correlation with functional motor outcome. MEPs are interpreted as an all-or-none phenomenon with regard to baseline recordings. When MEP amplitude is lost, urgent intraoperative intervention can often result in restoration of the waveforms. Permanent loss of MEP tracings is associated most often with new postoperative motor deficits.

\section{Minimally Invasive Approaches}

Minimally invasive surgery for intradural tumors offers the potential benefits of less postoperative pain, less blood loss, and quicker recovery, and it prevents long-term instability from multilevel laminectomy while maintaining spinal structural integrity. ${ }^{5,6,9,11}$

Although there are several technical connotations to the words "minimally invasive," complete tumor removal with no morbidity remains the objective. Some authors have used the tubular retractor system to perform muscle splitting by serial dilation and to reach the target lamina. ${ }^{1,8}$ Nzokou et al. ${ }^{8}$ proposed that even for foraminal tumors, when using a tubular retractor the need for facetectomy is eliminated, which decreases the incidence of postoperative deformity and eliminates the need for adjunctive fusion surgery. Other authors ${ }^{11}$ used unilateral subperiosteal dissection to reach the desired bone. In this approach, the aim is to remove just a sufficient amount of bone in a trajectory that is perpendicular to the entry point, which could even mean removing the lower part of the upper lamina and the upper part of the lower lamina to achieve the dural exposure targeted to cover the extent of the lesion. During partial facetectomy, undercutting of the base of the spinous processes and contralateral lamina, oblique tilting of the operating table can be done to visualize the contralateral side of the spinal canal better. If the initial operative plan is selected carefully, rarely does a hemilaminectomy have to be converted into a full laminectomy. Extending the segments vertically up to 3 levels is also feasible with this technique, which might be difficult to do with a tubular retractor system.

\section{Surgical Outcomes}

Gross-total resection rates for IDEM tumors are higher than those for intramedullary tumors and have ranged from $80 \%$ to $99 \%$ in various studies. It is not surprising that this is the most important variable determining outcome and recurrence. Even patients with severe preoperative neurological deficits experience complete recovery after a good total excision and adequate rehabilitation. Patients with cervical and lumbar tumors seem to have better outcomes than those with thoracic tumors. We feel that the kyphotic curvature of the thoracic spine, steep angulation of the spinous process, relatively narrow width of the thoracic canal, and precarious blood supply that results from the presence of a watershed zone make dealing with these pathologies challenging. Mehta et al. ${ }^{7}$ observed that patients who presented with a ventrally located IDEM tu- mor in the upper thoracic spine (T1-4) had the highest rate of worsening postoperative neurological deficits. They attributed this observation to the high cord-to-canal ratio and the tenuous blood supply of the cord in this region. Huang et $\mathrm{al}^{4}{ }^{4}$ commented that in addition to the abovementioned factors, tumor size, adherence to the thoracic cord, and the pathology also might be important factors to consider when operating on thoracic IDEM tumors. An understanding of the anatomy of this region, appropriate localization, and meticulous tumor removal without any handling of the cord are paramount for achieving a good outcome for intradural tumors of the spinal cord.

Neurosurgeons familiar with minimally invasive procedures for degenerative conditions such as lumbar disc prolapse and those who are comfortable operating through tubular retractors may be better positioned to quickly acquire the skills to remove intradural tumors through a minimally invasive approach. However, this technique needs to evolve over time, and the learning curve may be quite steep.

\section{Complication Avoidance}

Patient selection, as outlined above, is the key to a good outcome. Determining the level of the exposure through some form of localization technique is also essential. Because the approach to these tumors is through a small bony and dural opening, significant internal decompression should be achieved, and the excision is most often performed in a piecemeal fashion. In toto excision of tumors should be avoided, because it can result in pressure injury to the surrounding neural structures. To avoid complications, it is essential that the surgeon not hesitate to convert to the traditional laminectomy approach at any stage in the surgery if excision of the tumor is found to be difficult for any reason and the surgery is getting unduly prolonged. Once tumor removal is complete, the emphasis should shift to meticulous dural closure, because improper closure can result in either a CSF leak or even pseudomeningoceles.

Although minimally invasive procedures are gaining in popularity, safety and efficacy are primary goals when removing tumors of this nature, and the surgeon should choose an approach with which he or she is familiar to ensure a good outcome for the patient.

\section{Conclusions}

The hemilaminectomy approach to removing intradural tumors is quick and safe and results in minimal morbidity and no mortality. Preoperative MRI localization improves accuracy and reduces operating time by eliminating the need for intraoperative fluoroscopy.

\section{References}

1. Gandhi RH, German JW: Minimally invasive approach for the treatment of intradural spinal pathology. Neurosurg Focus 35(2):E5, 2013

2. Hirano K, Imagama S, Sato K, Kato F, Yukawa Y, Yoshihara $\mathrm{H}$, et al: Primary spinal cord tumors: review of 678 surgically treated patients in Japan. A multicenter study. Eur Spine J 21:2019-2026, 2012 
3. Hsu W, Sciubba DM, Sasson AD, Khavkin Y, Wolinsky JP, Gailloud P, et al: Intraoperative localization of thoracic spine level with preoperative percutaneous placement of intravertebral polymethylmethacrylate. J Spinal Disord Tech 21:72-75, 2008

4. Huang WC, Wu JC, Chang PY, Tu TH, Yen YS, Cheng H: Complication avoidance in intradural extramedullary spinal tumors. J Neurosurg Spine 20:768-769, 2014

5. Lu DC, Chou D, Mummaneni PV: A comparison of miniopen and open approaches for resection of thoracolumbar intradural spinal tumors. J Neurosurg Spine 14:758-764, 2011

6. Lu DC, Dhall SS, Mummaneni PV: Mini-open removal of extradural foraminal tumors of the lumbar spine.J Neurosurg Spine 10:46-50, 2009

7. Mehta AI, Adogwa O, Karikari IO, Thompson P, Verla T, Null UT, et al: Anatomical location dictating major surgical complications for intradural extramedullary spinal tumors: a 10-year single-institutional experience. J Neurosurg Spine 19:701-707, 2013

8. Nzokou A, Weil AG, Shedid D: Minimally invasive removal of thoracic and lumbar spinal tumors using a nonexpandable tubular retractor. J Neurosurg Spine 19:708-715, 2013

9. O'Toole JE, Eichholz KM, Fessler RG: Minimally invasive approaches to vertebral column and spinal cord tumors. Neurosurg Clin N Am 17:491-506, 2006

10. Paolini S, Ciappetta P, Missori P, Raco A, Delfini R: Spinous process marking: a reliable method for preoperative surface localization of intradural lesions of the high thoracic spine. Br J Neurosurg 19:74-76, 2005

11. Sim JE, Noh SJ, Song YJ, Kim HD: Removal of intraduralextramedullary spinal cord tumors with unilateral limited laminectomy. J Korean Neurosurg Soc 43:232-236, 2008

12. Slotty P Jr, Kröpil P, Klingenhöfer M, Steiger H-J, Hänggi D, Stummer W: Preoperative localization of spinal and peripheral pathologies for surgery by computed tomography-guided placement of a specialized needle system. Neurosurgery 66:784-787, 2010

13. Turel MK, Rajshekhar V: Magnetic resonance imaging localization with cod liver oil capsules for the minimally invasive approach to small intradural extramedullary tumors of the thoracolumbar spine. J Neurosurg Spine 21:882-885, 2014

\section{Author Contributions}

Conception and design: Rajshekhar. Acquisition of data: Turel, D'Souza. Analysis and interpretation of data: all authors. Drafting the article: Rajshekhar, Turel. Critically revising the article: Rajshekhar, Turel. Reviewed submitted version of manuscript: all authors. Approved the final version of the manuscript on behalf of all authors: Rajshekhar.

\section{Correspondence}

Vedantam Rajshekhar, Department of Neurological Sciences, Christian Medical College, Vellore 632 004, Tamil Nadu, India. email: rajshekhar@cmcvellore.ac.in. 